hielten sie diesen $z w a r$, doch weigerten sich die Bauern, sich weiterhin zu Arbeiten für die Häuptlinge heranziehen $\mathrm{zu}$ lassen (S. 209). An die Stelle der Vorstellung, mit der Arbeit für den Häuptling einen persönlichen oder kommunalen Dienst zu leisten, trat die Motivation individuellen Nutzens durch den Verdienst (S. 218). Wenn auch die persönliche Ausgestaltung des Verhältnisses zwischen dem einzelnen und dem Häuptling bis in die Gegenwart hinein erkennbar ist, so nahm sie doch beständig ab. In alter Zeit pflegten die Häuptlinge ihren Leuten für geleistete Arbeit ausdrücklich zu danken; unter dem Einfluß Europas schwand diese Sitte und machte der Entlohnung in Geld Platz (S. 218). Kennzeichnend für die Entwicklung ist die Ausweitung von Lohnarbeit und Handel auf Kosten der Gemeinschaftsarbeit mit anschließender Verteilung bzw. Benutzung der Produkte (S. 217).

Während es im alten Buganda nur wenige reine Berufsgruppen gab, gehörte ihnen seit den zwanziger Jahren dieses Jahrhunderts der größte Teil der männlichen Ganda an (S. 253). Neben den Berufen, die auf den durch europäischen Einfluß veränderten Bedürfnissen der Ganda beruhten, blieben auch manche der traditionellen Berufsgruppen unverändert erhalten. Die nomadisierenden Hima blieben weiter auf die Rinderhaltung spezialisiert (S. 249). Die einheimischen Schmiede konnten mit den europäischen Produkten konkurrieren, weil viele Gandafrauen glaubten, daß bei Benutzung fremder Werkzeuge die Saat nicht gedeihen würde (S. 261). Dagegen zogen die seit Beginn dieses Jahrhunderts immer zahlreicher nach Buganda einströmenden Asiaten, besonders Inder, den Handel weitgehend an sich; den Ganda fehlten hier die erforderlichen Kenntnisse, da die Missionare und Protektoratsbeamten insoweit keine Ausbildung vermittelten. Diese Entwicklung hat bis in die Gegenwart fortgewirkt und stellt noch heute die Regierung vor große Probleme.

Jensen weist nach, daß viele der gegenwärtigen sozialen und ökonomi- schen Schwierigkeiten darauf beruhen, daß sich das moderne staatliche Ordnungsprinzip noch nicht voll durchgesetzt hat, die traditionellen Formen aber durch die Entwicklung den inneren Zusammenhang verloren haben (S. 215).

Neben einer gründlichen Behandlung der Arbeitsteilung im allgemeinen und in Buganda im besonderen bietet das Werk eine Fülle von Informationen und Nachweisen, die nicht nur für den Soziologen sondern auch für den $\mathrm{Hi}$ storiker und Ethnologen von Interesse sind. Die Bedeutung, die die Entwicklung der afro-asiatischen Völker im Bewußtsein Europas zu erlangen im Begriff ist, sichert dem Werk die ihm gebührende Aktualität.

Klaus Peters

\section{Wolfgang Kaden}

\section{Das nigerianische Experiment}

Demokratie und nationale Integration in einem Entwicklungsland

Verlag für Literatur und Zeitgeschehen Hannover 1968

(Schriftenreihe des Forschungsinstitut der Friedrich-Ebert-Stiftung - Sozialwissenschaftliche Schriften)

186 S., $19,80 \mathrm{DM}$

Die wissenschaftliche und auch populärwissenschaftliche Literatur über die Verhältnisse in Nigeria hat in zehn Jahren eine bemerkenswerte Entwicklung durchgemacht: In den ersten Jahren stand eine euphoristische Schwärmerei über die bedeutende afrikanische Demokratie Nigeria im Vordergrund, die vor allem aus britischen Quellen gespeist wurde. In jüngster Zeit sind oft simplizierende Veröffentlichungen in den Vordergrund getreten, die das nigerianische Problem auf den Gegensatz muslimisch-christlich oder kommunistisch-westlich zurückführen wollen. Die deutsche Offentlichkeit ist im wesentlichen erst von dieser zweiten Welle der Nigeria-Literatur berührt worden, daher ist es besonders verdienstlich, wenn die Friedrich-Ebert-Stiftung mit der Veröffentlichung der Dissertation von Wolfgang Kaden eine materialreiche, gründliche Studie der nigeriani- 
schen Verhältnisse dem deutschen $\mathrm{Pu}$ blikum anbietet.

Die Arbeit ist mehr als die übliche, im wesentlichen auf die Ausschöpfung ausländischer Quellen und Beschreibungen gestützte, deutsche Dissertation, sie ist vielmehr eine originäre wissenschaftliche Arbeit, der man fast an jeder Stelle anmerkt, daß der Autor den Gegenstand seiner Forschungen in eigener Anschauung kennengelernt hat. Neben der gründlichen Darlegung der nigerianischen Probleme ergibt die Arbeit exemplarisch für die weitere Konzeption der deutschen Uberseeforschung den Beweis, daß die deutsche neben der angelsächsischen oder französischen Überseeforschung durchaus bestehen könnte, wenn man, wie in diesem Falle geschehen, nur den Mut und vielleicht noch eher die notwendige Einsicht findet, den interessierten Wissenschaftlern auch die Mittel für einen längeren Studienaufenthalt im Lande selbst zur Verfügung zu stellen.

Die Untersuchung von Kaden ist in drei Teile gegliedert, wobei die ersten beiden Teile das Gewicht des Buches ausmachen. Im ersten Teil gibt Kaden eine Analyse der Rahmenbedingungen, unter denen der Versuch des Bundesstaates Nigeria gemacht worden ist. $\mathrm{Er}$ beschreibt die ethnische und kulturelle Struktur Nigerias, das gesellschaftliche Gefüge und seine Wandlungen unter dem Einfluß der britischen Kolonialherrschaft. Am Ende dieser Beschreibung steht die Feststellung, daß im Grunde zwischen den vielen weit auseinandergezogenen Teilen des Landes und ihrer Bevölkerung keine hinreichende Kommunikation bestanden hat, so daß man schwer von gemein-nigerianischen Ideen sprechen kann, auf die der nigerianische Staat hätte aufgebaut werden können. Nach einer kurzen Abhandlung der Kolonialgeschichte gibt der Autor einen Úberblick über die erste Bundesverfassung und wendet sich dann im zweiten Teil dem politischen Geschehen in Nigeria zu.

Im zweiten Teil beschreibt der Autor zunächst die Geschichte der verschiedenen politischen Parteien und gelangt dabei zu dem Ergebnis, daß es sich primär um Parteien der großen Stämme gehandelt habe. Danach wendet er sich den Auseinandersetzungen zwischen den Parteien zu, dem Kampf zwischen der Action Group und dem Northern Peoples Congress sowie der Stellung des National Council of Nigeria and the Camerouns. Besonders befaßt er sich mit den Wahlen, vor allem der letzten Wahl vor dem Umsturz in Nigeria.

In dem dritten abschließenden Teil des Buches unternimmt der Autor den Versuch, auf Grund des von ihm dargestellten Bildes der nigerianischen Entwicklung nun den nigerianischen Bürgerkrieg $\mathrm{zu}$ analysieren und die auslösenden Faktoren herauszufinden. Seine erste These lautet, daß die Bundesstaaten $\mathrm{zu}$ groß konzipiert worden sind und bei dieser Konzeption dem Norden ein unverhältnismäßig starkes Gewicht gegeben worden ist (S. 152). Diese These deckt sich mit den Ansichten entscheidender nigerianischer Politiker, auf die der Rezensent schon bei früherer Gelegenheit in dieser Zeitschrift hinweisen konnte (Jahrgang 1968 , S. $41 \mathrm{f}$. und S. 235). Kaden begnügt sich allerdings nicht mit einer monokausalen Erklärung für das nigerianische Dilemma, sondern sucht nach weiteren Gründen. Auf Grund seiner Untersuchungen gelangt er zu dem Ergebnis, daß in Nigeria ein Konsens über die Systemnormen der Verfassung weitgehend gefehlt hat, womit die im ersten Teil bemängelte schlechte Kommunikation zwischen den Bevölkerungsgruppen in ihrer Bedeutung für die Entwicklung unterstrichen wird. Er sieht einen weiteren Grund für das Scheitern der ersten Verfassung darin, daß die Ibos ihren Bildungsvorsprung recht bedenkenlos ausgenutzt haben; aber nicht nur die Ibos sind in seinen Augen an dem Dilemma schuld, sondern auch die anderen Stämme, die fast allesamt in der Politik und den staatlichen Ämtern in erster Linie ein Vehikel gesehen haben, um für sich, aber auch für ihre Familie und ihren Stamm, materielle Vorteile zu erzielen. 
Insgesamt erklärt der Autor dieses Fehlverhalten aus einer mangelnden wirtschaftlichen Entwicklung des Landes (S. 156 ff.). Schon in dem ersten Absatz des Buches hat der Autor die Wirtschaftsentwicklung als den entscheidenden Motor der Entwicklung zum modernen demokratischen Staat bezeichnet. Der aus diesem Ansatz zu ziehende Schluß, den der Autor sich allerdings versagt hat, wäre, daß durch umfassende Wirtschaftsentwicklung, vor allem aber auch Entwicklungshilfe, das nigerianische Problem zu lösen ist. Der Rezensent kann nicht verschweigen, $\mathrm{da} ß$ er an dieser Grundthese trotz aller Freude über das Buch $Z_{\text {weifel hat, ob- }}$ wohl er meint, daß es hier im Grunde um die Frage geht, ob die Henne oder das Ei zunächst da war. Der Autor stellt auf ein Primat der Wirtschaftsentwicklung $a b$, der Rezensent meint hingegen, daß die Wirtschaftsentwicklung nicht Motor, sondern Parallelentwicklung zur politischen Entwicklung in Europa gewesen ist, und daß beide Entwicklungen ihren eigentlichen Ausgangspunkt für Europa in der beginnenden Rationalität und Rationalisierung des Lebens seit der Renaissance haben, daß sie in der folgenden Zeit in einem eng verflochtenen Prozeß sich gegenseitig vorangetrieben haben. Der Rezensent meint also, daß die Wirtschaftsentwicklung, die in einem Zeitalter der Entwicklungshilfe zunächst das Interesse findet, nicht das Allheilmittel ist und daß ein bloßes Abstellen auf die Wirtschaftsentwicklung den Weg zur Erkenntnis der nigerianischen Probleme im Grunde verstellt. Es ist $\mathrm{zu}$ fragen, ob nicht unter anderem auch ein unterschiedlicher Grad der Adaptations- und Rezeptionsbereitschaft für europäische rationale, $d$. h. nun einmal auch politische und wirtschaftliche Vorstellungen in Nigeria eine der Ursachen für die unglückliche Geschichte des Landes gewesen ist. Die Entwicklung Nigerias legt den Schluß nahe, $\mathrm{da} \beta$ die alten vorkolonialen Staaten Nordnigerias mit ihrer gefestigten Herrschaftsstruktur dem europäischen Denken viel mehr Widerstand entgegengebracht haben als etwa die sozial wenig strukturierten Teile des Ostens, deren Ibobevölkerung sehr schnell das europäische Denken adaptiert hat und damit den vom Autor dargestellten Bildungsvorsprung gewinnen konnte. Wenn der Rezensent auf dieses Problem hinweist, ist das nicht als Kritik an dem Buch $\mathrm{zu}$ verstehen, sondern als Anregung, bei einer weiteren Auflage in dieser Richtung noch einiges nachzutragen. Der Rezensent wünscht dem Buch eine baldige zweite Auflage und eine große Verbreitung.

Hinsichtlich der Lösung des nigerianischen Problems entläßt der Autor den Leser mit Zweifeln. Er ist skeptisch, ob eine Wiedervereinigung Nigerias bei einem Sieg des Bundes dauerhaft sein wird (S. 171). Als Hoffnungsschimmer läßt der Autor den „geographical frame" aufleuchten (S. 172). Ein Lösungsvorschlag für das nigerianische Problem war nicht das letzte Ziel des Buches, sondern es will das Verständnis wecken, das sollte ihm in Deutschland jedenfalls gelingen.

Dieter Schröder 